TRABAJOS DE PREHISTORIA

52, n. ${ }^{\circ} 2,1995$, pp. 31-45

\section{EL REGISTRO PALEOCLI- MÁTICO PLEISTOCENO EN LA EVOLUCIÓN DEL KARST DE ATAPUERCA (BURGOS): EL CORTE DE GRAN DOLINA}

\author{
PALEOCLIMATE RECORD OF \\ PLEISTOCENE IN THE EVOLUTION \\ OF ATAPUERCA KARST (BURGOS): \\ «GRAN DOLINA»SECTION
}

\section{MANUEL HOYOS (*) \\ EMILIANO AGUIRRE (*)}

\section{RESUMEN}

Se analizan las características sedimentológicas de los niveles del corte de Gran Dolina (TD) en la Trinchera de Atapuerca (Burgos) y el clima que se deduce, referenciando el contenido faunístico y arqueológico de cada nivel. Se indican las bases para la datación geocronométrica, así como la correlación de las fluctuaciones climáticas inferidas en Gran Dolina con los episodios $\mathrm{O}^{18} \mathrm{del}$ océano, contrastando aquéllas con evidencias paleontológicas. Se consideran en particular las fluctuaciones en la humedad y régimen pluvial.

\section{ABSTRACT}

Sedimentological characteristics of the different levels from the Gran Dolina (TD) section in the Atapuerca Cut (Burgos) are analyzed and climatic conditions inferred, with reference to the faunal and archaeological contents. The grounds for geochronological dating are stated as well as the correlation of climatic oscillations inferred from

(*) Museo Nacional de Ciencias Naturales, CSIC. José Gutiérrez Abascal, 2. 28006, Madrid.

El artículo fue remitido en su versión final el 5-IX-95.
Gran Dolina with oceanic $\delta^{18} O$ stages. Oscillations of humidity and pluvial regime are considered specially.

Palabras clave: Pleistoceno. Karst. Sedimentología. Paleoclima. Geocronología. Atapuerca.

Key words: Pleistocene. Karst. Sedimentology. Paleoclimate. Geochronology. Atapuerca.

\section{INTRODUCCIÓN}

El sistema kárstico de la Sierra de Atapuerca se extiende por la mitad sur de este accidente geográfico, con vergencia al Sur y al Oeste. Las cavidades conocidas, restos de otras mayores, se hallan dentro de un área de medio kilómetro cuadrado en número de 38 (Martín Merino et alii, 1981), situadas entre las cotas de 1030 y 971 $\mathrm{m}$. Pertenecen a subsistemas kársticos o «aparatos kársticos» (en el sentido de Llopis Lladó, 1970) diferentes, debido a condicionantes fundamentalmente tectónicos, ya que los estructurales y litológicos son comunes a todos ellos. Muchas de las cavidades censadas en esta zona se conocen por cortes en canteras y por los de la trinchera de un ferrocarril desmantelado (Fig. 1). Entre estos subsistemas kársticos destacan los siguientes: 


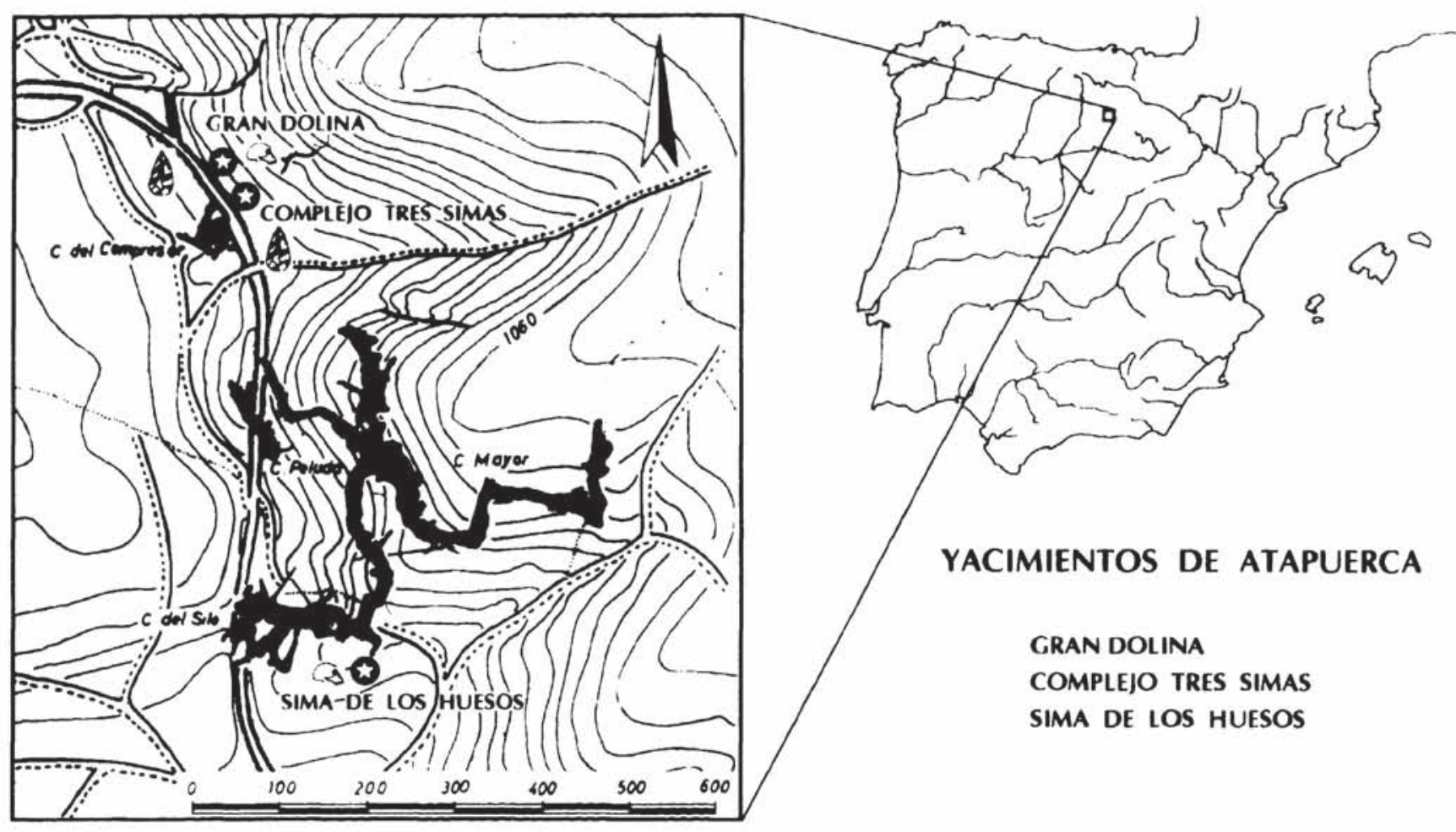

Fig. 1. Mapa de situación. Localización de los yacimientos principales del karst de Atapuerca. Burgos. Se señalan en negro las proyecciones de las cavidades actuales conocidas.

- Aparato kárstico de Cueva Mayor-Cueva del Silo, al que corresponde el complejo de cavidades BU-IV-A, 1 y 2, de Martín Merino (Martín Merino et alii, 1981), que incluye la Sima de los Huesos excepcionalmente rica en restos fósiles humanos.

- Aparato del Complejo Tres Simas-Cueva de los Zarpazos ( $n .^{\circ} 4$ del citado censo), con conductos fosilizados por sedimentos y cuya entrada pudo estar relacionada con la Cueva de los Zorros (n. ${ }^{\circ} 27$ ).

- Aparato de Gran Dolina con las cavidades asociadas de El Penal y las residuales n.os 16, 17 y 18 , también fosilizadas por rellenos sedimentarios, prolongándose la primera por el interior del macizo calcáreo.

Dentro del interés general del karst de Atapuerca por el singular yacimiento de fósiles humanos preneandertalenses de la Sima de los Huesos, por el registro paleolítico desde conjuntos preachelenses hasta achelenses finales, y el diverso, largo y bien calibrado registro paleoecológico, es el corte de Gran Dolina el que ofrece una secuencia más completa de depósitos bien estratificados, con más niveles fértiles en fósiles e industria lítica, y los registros de mayor antigüedad en estos aspectos. Además, recientemente se han encontrado fósiles humanos asociados a industria, polen y a un complejo faunístico anterior al de Mauer, del que hasta ahora no se conocían fósiles humanos en Europa.

En el presente trabajo se analizan las características sedimentológicas y paleoclimáticas de los rellenos sedimentarios de Gran Dolina, señalándose los niveles con industria del Paleolítico inferior y restos humanos.

\section{LA SECUENCIA SEDIMENTARIA DE GRAN DOLINA Y SU INTERPRETA- CIÓN PALEOCLIMÁTICA}

En todas las secuencias se han distinguido unidades sedimentarias con características genéticas y deposicionales específicas, separadas por discontinuidades, aunque los hiatus representados por éstas no tengan, por otros criterios, un valor temporal amplio. Dichas unidades son susceptibles de dividirse a su vez en subunidades con caracteres sedimentarios particulares. Tam- 
bién se corrigen y reinterpretan los datos preliminares aportados anteriormente (Gil et alii. 1987), conservando en lo posible la nomenclatura dada entonces a los diferentes niveles.

Los depósitos más antiguos de las secuencias más amplias conocidas en el karst de Atapuerca se encuentran por el momento en la Gran Dolina (Aguirre, 1992; 1995). Este corte tiene una potencia visible desde el suelo de la trinchera a la parte superior de ésta de $18-19 \mathrm{~m}$, por lo que ahora puede considerarse como la serie tipo con la que correlacionar otros depósitos.

La parte basal del relleno se encuentra diferenciada en dos zonas, separadas por una prominencia calcárea intermedia de forma apuntada, cuyo ápice alcanza el nivel superior de TD-5. El análisis de conjunto de ambas zonas muestra como más representativa la situada en el flanco NW, por lo que nos referiremos a ella.

\subsection{Análisis global de la secuencia}

En un primer análisis de conjunto de las características de los rellenos de la Gran Dolina se pueden hacer las consideraciones siguientes:

Excepto los dos-tres metros terminales de la secuencia, el resto de los sedimentos se encuentran afectados por deformaciones de colapsos gravitacionales con hundimientos en las zonas central y marginal, más acentuados y con mayor desplazamiento y mezcla de sedimentos en las proximidades del contacto entre depósitos y paredes de la cavidad, sobre todo en el borde Sureste, al añadirse a estos procesos los también postdeposicionales de circulación vertical de agua por dicha zona.

En la evolución de la secuencia de Gran Dolina se han distinguido tres grandes fases kársticas (Fig. 2):

$\mathrm{F}_{1}$ - Una primera fase endokárstica a la que corresponden los sedimentos de los niveles TD1 y TD-2, relacionados con un sistema de cavidades internas, sin comunicación con el exterior por la vertical.

$\mathrm{F}_{2}$ - Una segunda fase de conducto abierto al exterior, con depósitos de cueva relacionados en su génesis con las condiciones climáticas exteriores (TD-3 a TD-9).

$\mathrm{F}_{3}$ - Una tercera fase exokárstica (dolina), a la que corresponden los niveles TD-10 a TD-11, que indican la apertura cenital de la cavidad en la vertical considerada, aunque lateralmente esta cavidad se prolongue hacia el interior del karst.
Las direcciones predominantes de aportes han estado también ligadas a las diferentes etapas morfokársticas de la cavidad:

- En los depósitos basales (TD-1 y TD-2) es difícil la determinación de las direcciones de flujo, como se verá más adelante.

- De TD-3 a TD-7 los aportes presentan direcciones preferentes o dominantes próximas a Oeste-Este, procedentes de lo que falta en la trinchera excavada, o de la zona denominada "El Pẹnal», en dirección casi contraria a la pendiente de la ladera actual, de donde se deduce que tenía que existir forzosamente una morfología que impidiera, por una parte, el acceso directo y normal de los depósitos de la vertiente, y por otra que condujese a su vez los sedimentos en dirección casi contraria a ésta. Dicha morfología sólo es explicable por la existencia de un conducto kárstico abierto al exterior, es decir, una cueva.

- Los depósitos de TD-8 y TD-9 presentan un cambio, con direcciones próximas a N-S y EW más relacionadas ya con aperturas cenitales y con la pendiente, indicadoras por tanto del comienzo de un mayor desmantelamiento del techo de la cueva, debido a la erosión producida por la evolución de la vertiente. Al menos una entrada queda ocluida temporalmente.

- Finalmente, en los niveles terminales TD10 y TD-11, las direcciones de los aportes en torno a la dirección Oeste-Este y la pendiente deposicional de los sedimentos indican, por una parte, que los aportes están ya directamente relacionados con la vertiente, y por otra que existía ya en esta zona una cavidad abierta al exterior que constituía una depresión en la ladera (dolina), que se prolongaba hacia el interior del karst por el conducto que constituía el drenaje principal, tanto en etapas anteriores como en ésta.

Dadas las características de los depósitos y las direcciones de los aportes, la morfología de la cavidad encajante de los sedimentos debe prolongarse por tanto, en forma de conducto endokárstico hacia el Este, hecho que podrá comprobarse en el futuro con las excavaciones que se están realizando.

\subsection{Características sedimentológicas y paleocli- máticas}

La serie estudiada corresponde en la base a la zona más septentrional. En ella se han distinguido las siguientes unidades (Fig. 2): 

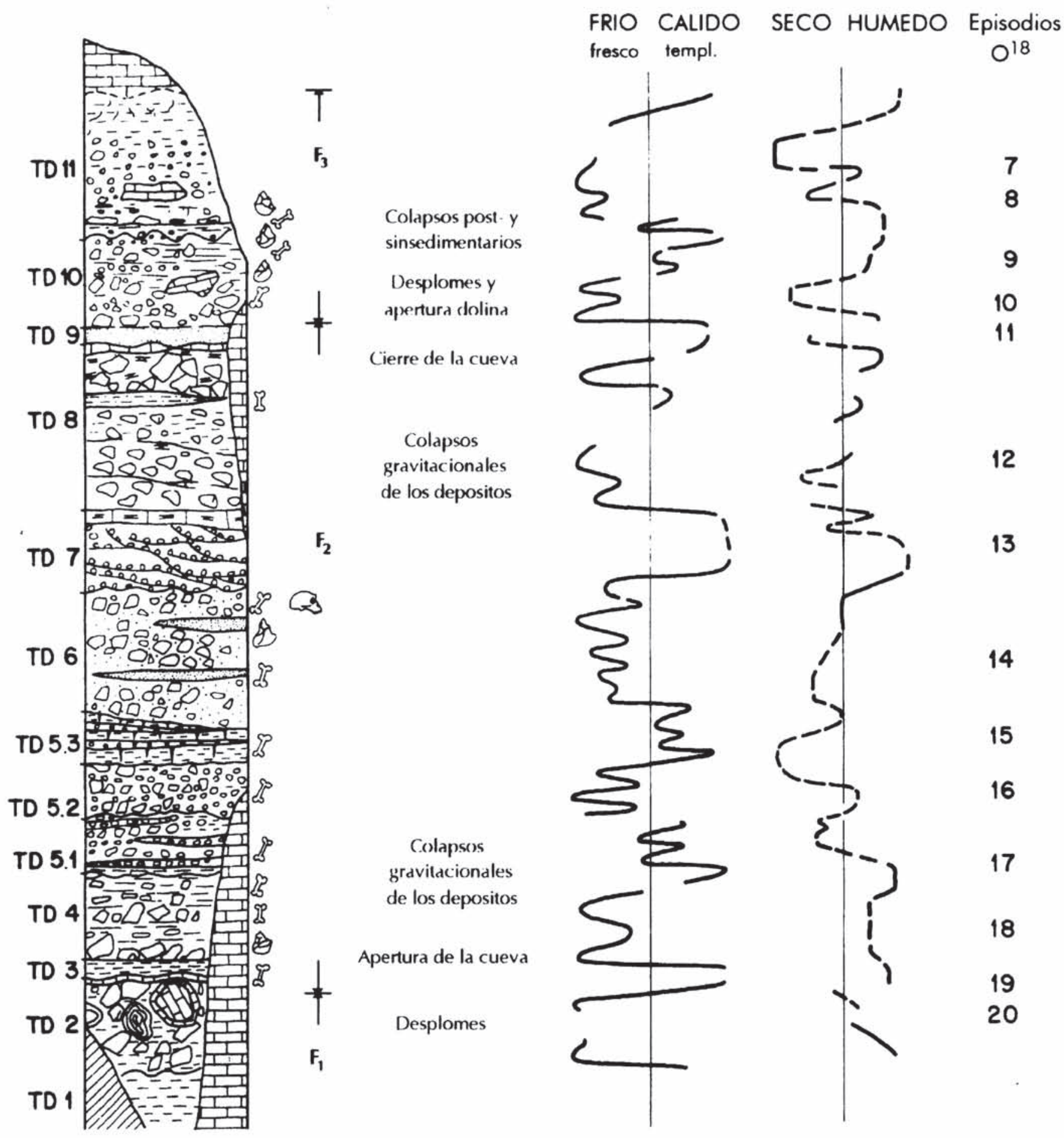

Fig. 2. Columna esquemática de la serie de depósitos de Gran Dolina, Atapuerca. Se indican los niveles con fósiles de vertebrados e industria; fases kársticas; curvas relativas de las variaciones de temperatura y humedad, señalando con trazo discontinuo las etapas de humedad estacional; correlación estimada con los episodios isotópicos del océano.

TD-1.- Potencia visible $0,5-0,6 \mathrm{~m}$. Aflora en la base del corte, presentando el techo deformado por carga y/o erosionado. Consiste en limos arenosos y arcillas de color verdoso masivas, de facies de inundación de una corriente hipogea de escasa capacidad de carga. Estos materiales son de origen endokárstico, procedentes de la disolución de las calizas encajantes en la zona interna del karst en ambiente no oxidante. Dicha corriente debería constituir o estar próxi- 
ma al freático local, marcando el límite entre las zonas freática y vadosa del karst en ese punto y en ese tiempo. Ello implica también que no existía en ese momento conexión con el exterior por la vertical de la cavidad.

No es posible una interpretación paleoclimática de estos depósitos dado su aislamiento del exterior cuando se generaron, ni siquiera establecer su relación con la humedad en función de los aportes de agua de infiltración, debido a su posición límite o próxima entre las zonas vadosa y freática del karst.

TD-2.- Potencia variable, máximo $1,6 \mathrm{~m}$. Se distinguen dos subunidades TD-2.1 y TD-2.2 de características diferentes:

TD-2.1.- Subunidad inferior compuesta por bloques heterométricos, algunos de gran talla, cantos y gravas calcáreas angulosas escasamente evolucionadas y restos de espeleotemas parietales y/o cenitales englobados en una matriz arcilloso-arenosa de color marrón rojizo; masivo y caótico, más escaso de matriz en la parte superior por lavado posterior. Corresponden a desprendimientos gravitacionales del techo de la cavidad, no necesariamente de la vertical de su situación actual, mezclados con aportes de finos por aguas de infiltración. Estos últimos proceden de la zona exokárstica y/o de rellenos previos de conductos y fisuras que desembocasen en la cavidad.

La atribución de estos depósitos a procesos de gelivación es arriesgada y no parece ser la más apropiada ya que falta en el conjunto de los detríticos gruesos alguna de las fracciones canto y son muy escasas las plaquetas. No se han encontrado criterios suficientes para su atribución a procesos sísmicos o tectónicos, por lo que parece más razonable atribuir estos desprendimientos a evolución cenital de la cavidad con colapso parcial del techo y apertura de una o más comunicaciones con el exterior. Quizá estén relacionados también con actividad biológica de raíces que ampliarían fracturas y diaclasas en el sustrato calcáreo.

TD-2.2.- Una segunda etapa sedimentaria está representada por una corteza estalagmítica pavimentaria cíclica, constituida por cristales de calcita en empalizada, de escasa potencia (máximo $4-5 \mathrm{~cm}$ ) que recubre el depósito anterior, con raíces de la misma y carbonataciones parciales en los huecos y fisuras.

La formación de este depósito se debe exclusivamente a aportes de aguas bicarbonatadas en régimen laminar con la práctica ausencia de detríticos. Este tipo de espeleotema se genera siempre en ambiente endokárstico, por lo que es factible que una eventual apertura al exterior de la cavidad generada en la etapa anterior se situase alejada de la vertical de la misma, y por tanto el depósito infrayacente deba su situación a rodamiento y/o deslizamiento inmediato o posterior a su llegada al suelo por el desplome.

Este tipo de espeleotema se asocia generalmente a clima templado y seco en el área mediterránea. En este caso y pese a la posición endokárstica de la corteza, la asociación a un clima seco, con lluvias estacionales, es admisible, ya que por comparación con el resto de los niveles de la serie, en los que el agua es un factor importante en el transporte de los sedimentos, es necesaria para la formación de ésta una disminución del régimen de circulación del agua hasta aportes laminares y/o cenitales de aguas carbonatadas y sin detríticos. Por el contrario, la asociación de dicha corteza con un clima templado o cálido, no es tan clara en este caso por la situación mencionada de la misma, sin embargo la ausencia de indicadores de clima frío, como los que existen en otros puntos de la serie, permite por exclusión relacionar esta costra con un clima benigno.

TD-3.- Potencia máxima $0,30 \mathrm{~m}$. Arcillas marrón rojizas compactas con un cierto tendido paralelo, aunque deformado por colapso, marcado por algunos lechos discontinuos de orden centimétrico de detríticos arenoso-limosos con alguna grava calcárea alterada en superficie y abundantes restos fósiles de microvertebrados, probablemente procedentes de la disgregación y arrastre de restos de aegagrópilas de la zona próxima al exterior, que se han comportado como clastos. Corresponden con aportes esporádicos de aguas de escorrentía no canalizadas y de escasa competencia, procedentes del exterior.

Dada la relación de este nivel con el exterior. y la ausencia de indicadores de clima frío y el grado de alteración de los elementos gruesos (gravas), se interpreta este nivel como generado en condiciones climáticas templadas y más húmedas que las del nivel anterior. Contiene fósiles de macro y micromamíferos (Aguirre, 1995, con referencias).

TD-4.- Potencia variable 1,1-1,3 m. Contacto con el anterior deformado y erosivo. Depósito muy heterométrico de cantos y bloques de caliza angulosos y frescos o con escasa alteración, 
algunos con fracturación secundaria por gelivación en el suelo. con matriz arenoso-arcillosa amarillento-grisácea. Presenta estratificación en lechos irregulares de orden decimétrico escasamente estructurados interiormente. con cantos y bloques más abundantes en las zonas centrales. con aspecto masivo y caótico, y mayor abundancia de finos hacia los extremos. El conjunto aparece deformado a su vez por colapso y deslizamiento.

Los procesos de gelivación. de acusada intensidad en relación con otros existentes en la secuencia, son las características fundamentales en la génesis de este depósito. Dichos procesos son más importantes en la base y tramo central con una pulsación menor entre ambas fases, disminuyendo posteriormente a techo. Están acompañados de finos aportados por aguas de escorrentía en las etapas de deshielo, deslizando en conjunto al interior de la cavidad a lo largo de diferentes etapas de solifluxión.

Por lo anteriormente expresado, estos depósitos se asocian a un clima frío, más riguroso en la base y tramo central, más benigno hacia el techo. Mientras, la humedad pasa de condiciones poco húmedas en los tramos basal y medio a condiciones más húmedas a techo.

Antes de la sedimentación de la unidad siguiente (TD-5), en el flanco NW se produce el colapso y deslizamiento de parte de esta unidad y depósitos infrayacentes, generándose una pequeña depresión que será rellenada por los sedimentos basales de TD-5. Contienen abundantes fósiles de mamíferos (Aguirre, 1995), sobre todo en la parte superior, y artefactos líticos del Paleolítico inferior a techo (Mosquera y Carbonell, 1992).

TD-5.- Potencia variable, máximo 3,35-3,40 $m$ en el lado NW y mínimo 1,40-1,50 en el SE. Se trata de una unidad compleja en la que se distinguen cuatro subunidades de características genéticas y sedimentarias diferentes.

TD-5.1.- Corresponde a la subunidad inferior, con una potencia 1,10 a $0,75 \mathrm{~m}$, mayor en la zona NW del corte. Está formada por una alternancia de niveles de dos tipos que se repiten constituyendo secuencias producidas por idénticos procesos sedimentarios aunque de diferente intensidad. Las facies corresponden a lo siguiente:

A) Por una parte se encuentran tres niveles de conglomerado masivo o pobremente estructurado con un cierto tendido paralelo a la estra- tificación general. Están compuestos de cantos y gravas calcáreas angulosas. con dos «stocks" en función de la alteración, unos escasamente alterados y otros, mayoritarios, bastante más alterados, por ser en parte heredados del tramo infrayacente, todo ello englobado en una matriz arenoso-arcillosa de color rojo.

Estas facies corresponden a flujos de barro que incluyen cantos de gelivación heredados de etapas anteriores y otros debidos a procesos de gelivación contemporáneos y de escasa intensidad.

B) Por otra parte, alternando con los anteriores, se aprecian tres niveles, el primero en la base, uno intermedio y otro finalmente a techo, constituidos por microconglomerado de potencia decimétrica $(0,25$ a $0,08 \mathrm{~m})$ de gravas y gravillas calcáreas con algunos cantos y plaquetas dispersos, y matriz arenosa-arcillosa, a veces muy escasa por lavado posterior. Presentan estratificación interna paralela, y la general adaptándose inicialmente a la morfología de la cubeta preexistente. Son ligeramente erosivos sobre los anteriores en la zona SE; el último de estos niveles erosiona el techo de TD-4.

Corresponden estos niveles a aportes de aguas de escorrentía pobremente organizada en pequeños canales planos de régimen discontinuo y con poco espesor de lámina de agua.

La secuencia tipo está formada por la asociación de facies B-A. En ella, a los aportes de los microconglomerados (B) les siguen fases de lavado superficial de la zona externa e incluso de los depósitos previos recién sedimentados y de los fangos, que dan lugar a las facies $\mathrm{A}$.

Se interpretan estos depósitos como formados en una alternancia de pulsaciones climáticas que comenzarían (Facies B) por unas condiciones climáticas «frescas», (algo más frías que las actuales Hoyos, 1979; 1981), en las que se producirían algunas heladas invernales a veces de cierta intensidad, con unas condiciones poco húmedas. A éstas les siguen pulsaciones de clima más templado en el que las heladas invernales siguen existiendo pero los procesos a los que dan lugar son aún menos importantes que en los anteriores (Facies A). Por su parte la humedad aumenta de $\mathbf{B}$ a $\mathbf{A}$, donde además las lluvias son más estacionales. Contienen fósiles de pequeños vertebrados.

TD-5.2.- El contacto con la subunidad anterior es ligeramente erosivo. Potencia variable de $1,70-1,60 \mathrm{~m}$ en la zona NW y $1,20-1,10 \mathrm{~m}$ en la 
zona meridional. Está constituido por un conjunto de dos cuerpos conglomeráticos en forma de cuña procedentes del $\mathrm{W}$ con una intercalación de detríticos más finos.

Los cuerpos conglomeráticos inferior y superior, corresponden a conglomerados muy heterométricos de cantos y gravas calcáreas con algunos bloques englobados en una matriz arcilloso-arenosa de color marrón rojizo. Los detríticos gruesos son angulosos o subredondeados con una cara fresca, algunos presentan fracturación secundaria por hielo en el suelo y son escasos los heredados. La estructuración interna es masiva, caótica, con los ejes mayores de los cantos en diferentes posiciones del espacio, siendo más desorganizada aún en el inferior.

Se interpretan como lóbulos de deslizamiento en masa, correspondiente el inferior, por su geometría, más a una colada de barro que de solifluxión, ya que acaba de forma brusca en un amontonamiento caótico de cantos, y el superior a una colada de solifluxión, aunque la terminación en forma más acuñada se debe a procesos erosivos posteriores de lavado lateral por circulación de agua superficial. Ambos transportan clastos de gelivación de procesos en su mayor parte coetáneos con los respectivos depósitos.

La intercalación de detríticos más finos está compuesta por gravas, plaquetas medianas y gravillas calcáreas con matriz arcilloso-arenosa marrón rojiza, con una cierta estratificación paralela en lechos discontinuos marcada por alineaciones de los elementos más gruesos.

Corresponden estos depósitos a aportes esporádicos de aguas de escorrentía laminares, no canalizadas, de baja energía y capacidad de carga, procedentes del exterior, con las mismas direcciones de aporte que los lóbulos citados. Rellenan en primer lugar la zona deprimida generada en su parte distal por la geometría del lóbulo inferior, recubriéndolo posteriormente y regularizando la superficie del suelo.

Estos depósitos se interpretan como correspondientes a dos pulsaciones de carácter frío y húmedo, separadas por un intervalo templado y húmedo. La primera pulsación sería algo más cruda que la segunda, con heladas más intensas. Por su parte el depósito intermedio correspondería con un clima templado en el que no desaparecerían por completo las heladas en la estación invernal, como indica la presencia de plaquetas, aunque aquéllas serían escasas y poco intensas. Contiene fósiles de pequeños vertebrados (Aguirre, 1995).

TD-5.3.- La potencia disminuye suavemente de $0.60 \mathrm{~m}$ en la zona NW a $0,35-(0,40 \mathrm{~m}$ en el SE. Entre este tramo y el anterior se registra una fase erosiva importante que da lugar a la excavación de un "canal» en artesa de base plana, conservándose la cicatriz de uno de sus bordes en el flanco SE, por lo que esta subunidad se sitúa en discordancia erosiva sobre la anterior.

Está compuesta por dos niveles de limos arcillosos y arcillas rojizas con cierta disyunción prismática en los que se intercala un nivel con base erosiva sobre el inferior, compuesto de gravillas bastante homométricas y arenas calcáreas, con predominio de las formas subredondeadas y con cierta alteración superficial. La matriz limoso-arcillosa de este último es análoga a los limos de los niveles encajantes, siendo escasa en algunas zonas por lavado.

El nivel inferior se interpreta como los aportes finales de fracciones finas con predominio de procesos de decantación, debidos a pérdida de energía de la corriente de agua que produjo la formación del canal citado. La disyunción prismática y los escasos precipitados de óxidos de hierro y manganeso se atribuyen a ciclos de humectación-desecación, con un cierto aspecto pedogenético aunque la génesis no es propiamente edáfica.

El nivel de gravillas corresponde a una fase de reactivación de la circulación hídrica, con aportes por aguas ocasionales de mediana a baja energía.

El nivel superior presenta características deposicionales análogas al inferior, aunque es más extenso, fosilizando ya el ápice del espolón calcáreo citado al principio, con mayor potencia de depósito y con algún lecho discontinuo de detríticos más gruesos (gravilla-arena).

Todo el conjunto se interpreta como asociado a un clima templado-cálido y seco, menos. húmedo que en la etapa anterior, con tendencia a una cierta aridez, en el que las escasas precipitaciones tuvieron un marcado carácter estacional, aunque ocasionalmente pudieron ser más violentas de forma local. Hay fósiles de pequeños vertebrados (Aguirre, 1995).

TD-5.4.- Corresponde al tramo final de esta unidad, con potencia variable de $0,00 \mathrm{~m}$ a 0,50 $0,60 \mathrm{~m}$ en el flanco NW, siendo erosivo el contacto con TD-5.3 y estando a techo erosionado parcialmente por la base de la unidad siguiente. 
Esta subunidad está formada por fangos de color marrón claro, que empastan abundantes gravas calcáreas angulosas y subangulosas con algunas plaquetas y cantos de la misma naturaleza y pequeña talla. las primeras más frescas que las de la unidad infrayacente. La estratificación es predominantemente masiva con alguna alineación poco definida de elementos gruesos en diferentes posiciones del espacio. A techo en la zona SE. aparecen restos de carbonataciones de origen diagenético.

Se interpreta como un depósito formado por aguas de escorrentía no canalizadas en régimen laminar que incorpora elementos calcáreos procedentes de procesos de gelivación de poca entidad. Corresponden a unas facies intermedias, que pueden representar la transición entre las del techo de TD-5.3 y las que le siguen a continuación, TD-6.

Desde un punto de vista paleoclimático representan el inicio de un cambio hacia condiciones frías y más húmedas en relación a la subunidad anterior, donde comienzan a sentirse los efectos de las heladas y un aumento de la humedad, que se caracteriza sobre todo por no ser ya estacional.

TD-6.- Potencia 2,40-2.10 m, ligeramente superior en el flanco NW. Es una unidad también compleja cuya base es erosiva sobre la infrayacente y su techo está erosionado por la siguiente TD-7. Estos depósitos llegan en su parte superior a fosilizar el resalte NW en forma de escalón que debía corresponder en ese momento al suelo de la cavidad, apoyándose y extendiéndose más ampliamente por dicha zona.

En conjunto se trata de un depósito constituido fundamentalmente por conglomerados calcáreos en los que se distinguen hasta cinco episodios de cuerpos conglomeráticos de secciones irregulares y diferente potencia, donde los elementos más gruesos se sitúan en la zona central de forma caótica, en todas posiciones y con escasa matriz, aumentando el tamaño y la abundancia de éstos en la vertical hasta el cuarto episodio. Los bloques y cantos presentan aristas frescas y superficie poco alterada, siendo escasos los elementos heredados. Hacia los flancos la matriz es más abundante y el tamaño de los cantos disminuye, tendiendo la estratificación a ser paralela, marcada por lechos discontinuos de cantos y gravas cuyos ejes mayores se sitúan en posiciones más estables. Entre el tercero y cuarto episodios se detecta una discontinuidad erosi- va previa a la sedimentación de este último, donde la distribución interna de los materiales presenta una mayor organización paralela.

La génesis de estos depósitos está relaciona. da con aportes gravitacionales de importantes productos de gelivación, a los que se suman aportes por aguas de escorrentía no canalizada durante las épocas de deshielo. Los procesos de gelivación presentan pulsaciones que crecieron en intensidad en la vertical hasta la penúltima de estas pulsaciones, decreciendo ligeramente en la última. Los aportes hídricos son de características similares en toda la unidad excepto en el momento correspondiente a la discontinuidad, donde debieron de ser más intensos erosionando y redistribuyendo los materiales más superficiales y regularizando la superficie del suelo; también durante el quinto episodio sedimentario, donde los aportes de finos y la regularización y distribución tendente a la paralela indican una mayor actividad de las aguas de escorrentía.

La falta de matriz y las recristalizaciones de carbonato existentes en algunos huecos entre los cantos, corresponden a lavados y precipitación de carbonatos postsedimentarios respectivamente, ocurridos de forma selectiva en función de la diferente permeabilidad de las capas y a favor de las discontinuidades entre éstas.

El conjunto de esta unidad se generó en un clima frío y húmedo, más crudo que los representados en etapas anteriores, marcado por cinco pulsaciones crecientes en la vertical, con un máximo en la cuarta y una ligera disminución en la última. Cada pulsación comienza por una fase menos fría y húmeda en la base, que da paso a condiciones más rigurosas y también húmedas a techo, para retornar de forma más rápida otra vez a un clima menos riguroso, inicio de una nueva pulsación. La última pulsación representa una regresión de las condiciones frías con respecto a la anterior; a la vez puede afirmarse que las condiciones de humedad por el contrario aumentan. Abundan los vestigios de fauna de vertebrados (Aguirre, 1995) y de industria lítica (Mosquera y Carbonell, 1992). Recientemente se han encontrado fósiles humanos en un tramo superior de esta unidad.

TD-7.- Presenta sección de canal acuñándose hacia el NW, con mayor espesor en la zona central de la cavidad dando una geometría convexa en la base, erosiva sobre el techo de la unidad infrayacente, con su techo plano, y una potencia en la zona central de 1,80-2,00 m. Está formada 
por conglomerados de gravas gruesas y arenas calcáreas y escasos cantos de pequeña talla. todos ellos redondeados o subredondeados, con escasa o nula matriz arcillosa y fuerte cementación carbonatada. Presenta estructuras internas hidrodinámicas con cuerpos sigmoidales y barras separadas por cicatrices erosivas, con estratificación cruzada, en surco y paralela. La dirección general de los aportes se sitúa próxima a Oeste-Este.

Se interpreta como un depósito fluvial de aguas torrenciales, asociado a fuertes y ocasionales lluvias, preferentemente tormentas, de un arroyo cuyo cauce sería de colector de las aguas superficiales de la vertiente y se sumía por la cueva. La carbonatación que presentan estos materiales es postsedimentaria, producida durante una etapa seca posterior no determinada.

La ausencia de indicadores de clima frío, teniendo en cuenta lo evolucionados que están los materiales gruesos y que ni siquiera se observa en ellos la presencia de plaquetas, siendo procedentes todos ellos de un área restringida de drenaje en la vertiente, y dado que si hubiese habido clastos generados en procesos de gelivación contemporáneos los habría incorporado igualmente el arroyo, nos inclina a asociar la génesis de esta unidad a un clima cálido, al menos templado. En cuanto a la humedad puede decirse que corresponde a un clima estacional, no necesariamente muy húmedo pero con lluvias importantes y cortas, de características parecidas a las actuales o más húmedas, con reducción posterior de la humedad.

TD-8.- La potencia observable en la zona NW de este depósito es variable, de 5,20 m en el extremo NW, donde alcanza prácticamente el techo de la cavidad existente todavía en esa zona, a 2,60 m hacia el centro, donde está fuertemente erosionada. Constituye una unidad compleja básicamente conglomerática, en la que se distinguen tres subunidades separadas por discordancias erosivas y susceptibles de subdividirse a su vez en niveles internos, así como una cuarta de características sedimentológicas diferentes, en la que por no variar la nomenclatura ya publicada mantenemos su denominación, añadiendo algunas correcciones necesarias.

TD-8.1.- Potencia de $1,50 \mathrm{~m}$ en la zona más al NW, y se acuña en el centro. Corresponde a un conjunto de tres acumulaciones sucesivas de lechos de bloques y cantos calcáreos bastante heterométricos, con predomino de los elementos frescos sobre los heredados, más redondeados: la matriz arenosa-arcillosa es escasa, a veces ausente por lavados postdeposicionales. Hay zonas o niveles de abundante carbonatación secundaria. Presentan un buzamiento aparente entre $10^{\circ}$ y $15^{\circ}$ con una dirección de aportes próxima a Norte-Sur, sensiblemente diferente a las direcciones dominantes en las unidades inferiores que procedían de la dirección Oeste. Los diferentes niveles son erosivos sobre los infrayacentes y progradantes en la vertical, extendiéndose progresivamente sobre el techo de la unidad anterior. La estructuración interna de los diferentes cuerpos presenta un cierto ordenamiento en el sentido de la sedimentación. En la zona NW, presentan deformación local por colapso, con la generación de una ligera depresión previa a la subunidad siguiente.

Este cambio en la dirección de aportes hacia el Norte y la disposición con buzamiento acusado de los depósitos implican un cambio en la morfología externa de la cavidad, al abrirse un nuevo acceso al exterior por el Norte, que evolucionará más tarde hacia lo que será una parte de la apertura cenital generalizada de la «Gran Dolina».

Estos depósitos corresponden a diferentes etapas de aportes de un cono de derrubios, retocados superficialmente por las aguas de escorrentía sobre todo los superiores. La presencia dominante de cantos frescos y la amplia heterometría de éstos implican la existencia de procesos de gelivación contemporáneos del depósito, procediendo los clastos tanto del exterior como de aportes gravitacionales de las paredes y techo de la zona externa de la cueva. La intensidad y duración de estos procesos no es tan acusada como en la unidad TD-6, incluso disminuye sensiblemente a techo, presentando un máximo relativo en la etapa intermedia. Por su parte, la progradación de los depósitos, los aportes de finos y la estructuración interna son más importantes en la etapa superior, donde las removilizaciones del techo del depósito y la erosión del mismo con la generación de una zona deprimida hacia el centro de la cavidad se manifiestan en el tenue ordenamiento de los cantos y gravas en la misma dirección de la superficie de erosión que se está generando.

Se interpreta esta subunidad como formada en unas condiciones climáticas frías crecientes hasta un máximo relativo en su zona central, 
para mejorar después a techo. A la vez. las condiciones de humedad aumentan progresivamente de muro a techo. pasando de un clima con unas características de humedad análogas o algo inferiores a las de la unidad precedente, a un clima más húmedo a techo con mayores aportes de agua.

TD-8.2.- Potencia $0.40 \mathrm{~m}$ a $1.20 \mathrm{~m}$. Es fuertemente erosivo sobre el anterior. Está formado por un conglomerado inicialmente fangoso plástico de cantos de caliza angulosos y subangulosos bastante frescos con matriz arcillosa rojiza y cementaciones carbonáticas secundarias en zonas preferenciales, que se adapta a la depresión existente en la zona central. La estructuración interna es masiva y caótica, presentando una geometría externa de forma lobular compuesta o polilobular coalescente.

Se interpreta este conjunto como lóbulos de solifluxión coalescente, tendentes principalmente hacia la zona central y procedentes de una dirección algo más virada al Este, próxima al $\mathrm{N}$ NE. El techo de esta subunidad se encuentra ondulado y presenta mayor proporción de plaquetas en la zona central, llegando en ella a faltar la subunidad siguiente TD-8.3 debido a la erosión posterior a esta última y previa a TD-8.4.

Corresponde esta subunidad con una fase fría y húmeda en la que por el momento no es posible distinguir episodios interiores. Puede considerarse como continuación más amortiguada de la fase fría de TD-8.1.

TD-8.3.- Potencia variable por estar fuertemente erosionado este tramo a techo. Estos depósitos llegaron a colmatar la apertura abierta por el flanco NW de donde procedían. Presenta las características conglomeráticas de los anteriores, aunque con tamaños de clastos menores y mayor abundancia de gravas y arenas.

Su interpretación sedimentológica y climática es análoga a la de las subunidades anteriores, precisando que en ésta la intensidad de los procesos de gelivación remitieron considerablemente con respecto a aquéllas, y la humedad aumenta con respecto a las mismas.

Posteriormente a esta unidad se producen una serie de procesos de erosión intensos sobre los materiales del techo, creándose sobre aquélla un paleorrelieve erosivo, ligado a la circulación de agua procedente del exterior que coinci- de con una fase húmeda importante. pero sin evidencias de clima frío.

TD-8.4.- A la formación de este paleorrelieve le sigue una etapa de carbonatación por aguas de infiltración que cementa los sedimentos de TD-8.3. Posteriormente. cuando el conjunto superficial es impermeable por la cementación citada, comienza el recubrimiento con la formación de una costra estalagmítica discontinua, generada por las mismas aguas ya en un régimen de circulación superficial, laminar y discontinuo, coincidente con una fase más seca y cálida. La costra no se extiende sobre toda la superficie de los depósitos precedentes, sino que se restringe a la zona noroeste extendiéndose hasta el centro.

La generación de este tipo de espeoleotema es un fenómeno normal sobre depósitos que colmatan el acceso exterior de una cueva, sea colmatación por relleno hasta el techo o por hundimiento de la cornisa de entrada (Hoyos, 1979; Hoyos et alli, 1982). En este caso, en el que sedimentos de TD-8.3 llegaron al techo y fueron luego parcialmente erosionados, caben dos posibilidades. La primera y más factible es que cayesen algunos bloques de la entrada y se obstruyese ésta por completo; la segunda sería que las dimensiones del acceso fuesen tan reducidas que los sedimentos exteriores quedasen retenidos al exterior, de modo que sólo penetrara el agua por infiltración, pero en este caso tendrían que encontrarse arcillas entre las laminaciones de la corteza. El registro paleontológico de TD-8 empieza a aumentar en la última campaña de excavación, destacando la presencia de hipopótamo.

TD-9.- Potencia media $0,25-0,30 \mathrm{~m}$, estando este nivel erosionado por la entrada de los materiales de la unidad siguiente. Está formado por arcillas limosas marrones, con manchas verdes de hidromorfismo en algunas zonas y marrones rojizas en otras. Engloba algunos cantos pequeños de caliza alterados. Presenta estratificación masiva, aunque en algunas zonas las discontinuidades internas están marcadas por nivelillos de carbonataciones finas secundarias.

Se interpreta como un depósito generado por aguas de escorrentía estacionales, que se infiltran por grietas y pequeñas aberturas en la zona de la antigua entrada. Corresponde con unas condiciones de clima templado o cálido y algo más húmedo que en la fase climática precedente. Se considera como la fase final de una amplia 
etapa interglacial cuyo comienzo podría estar en la fase de erosión sobre el techo de TD-8.

Entre TD-9 y TD-10 se produce una nueva apertura o ensanche por caída de bloques del techo de la mitad noroccidental de la cueva. Apertura y desmantelamiento del techo que continuará durante la sedimentación de TD-10. incorporando bloques a los sedimentos procedentes del exterior de esta unidad; algunos de aquéllos, de grandes dimensiones, sobrepasan ampliamente $1 \mathrm{~m}$. Puede afirmarse por tanto, que a partir de este momento la cueva, en la vertical del ámbito estudiado, comienza a perder su techo por hundimiento y la comunicación con el exterior se extiende progresivamente por toda la superficie de lo que conocemos actualmente como Gran Dolina, pasando a comportarse como una forma exokárstica. En dirección Nornoreste debía continuar la cueva hacia el interior del macizo calcáreo. Con esta disposición morfológica, la dolina actuaba como colector y la cueva, ya casi colmatada, como sumidero de las aguas y sedimentos detríticos.

TD-10.- Lo que en publicaciones anteriores (Gil et alii, 1987) llamamos TD-10 es una unidad detrítica compleja, susceptible de dividirse. Contiene evidencias de cambio de régimen, y de evolución notable de la cavidad en un nuevo ciclo de actividad kárstica ya muy tardío. Las direcciones de aporte son centrípetas y convergentes hacia el Este del corte expuesto, donde se sitúa el centro de la depresión que estaría comunicado horizontalmente con la cueva; ésta se prolongaría en esa misma dirección hacia el interior del macizo calcáreo, es decir actuaría como colector de aportes hacia lo que quedase de cueva. La pendiente deposicional, al principio más acusada, se amortigua paulatinamente en la vertical hasta completarse más tarde el relleno con la sedimentación de TD-11.

TD-10.1.- Con una potencia variable, que suele alcanzar y rebasar $0,75 \mathrm{~m}$. Está compuesta por dos niveles de cantos y gravas de caliza angulosos empastados en una matriz arcillosa, separados por un nivel más arcilloso rojizo que incorpora algunos cantos y gravas dispersos. Todos ellos proceden del NW dirigiéndose hacia el centro.

El primer nivel de cantos tiene forma lobular con la base ligeramente convexa que erosiona y se encaja en TD-9, con mayor potencia en el centro $(0,20-0,30 \mathrm{~m})$. Los cantos heterométricos $\mathrm{y}$ angulosos presentan ocasionalmente fractura- ción secundaria por hielo. La estratificación presenta una cierta tendencia a la organización interna en forma de lechos paralelos marcados por alineaciones discontinuas de cantos y plaquetas en sus posiciones más estables. Todo el conjunto se encuentra buzando hacia el centro de la depresión.

Se interpreta este nivel como una acumulación cíclica de lechos de cantos de gelivación con aportes escasos de finos por aguas de deshielo. Cuando el depósito alcanzó suficiente masa, deslizó como una colada densa a favor de la pendiente erosionando el nivel infrayacente y adquiriendo la forma lobular mencionada. Dicho transporte fue corto y no modificó prácticamente la estructuración interna primaria.

Corresponde con una pulsación fría y poco húmeda, en la que se dan fenómenos de gelivación en el suelo, aunque no importantes.

Le sigue un nivel de $0,12-0,15 \mathrm{~m}$ de arcillas rojas con cantos y gravas frescas, dispersos en su interior, con estratificación masiva, que se interpreta como un depósito generado por aguas de escorrentía no canalizadas, al que se incorporan algunos productos de gelivación, en el que las condiciones frías de antes remiten considerablemente pero sin desaparecer por completo, en un ambiente de mayor humedad.

En la parte superior de esta subunidad se sitúa un nuevo nivel de bloques, cantos y gravas calcáreos más heterométrico y con mayor proporción de matriz arcillosa rojiza que el primero, sin cantos con gelivación secundaria, y menos estructurado que el mismo. Sus características sedimentarias son análogas a las de éste, sin el carácter cíclico apuntado para el mismo. Debió generarse en un ambiente frío, con heladas más intensas y duraderas que las del nivel inferior.

En conjunto esta subunidad representa una fase fría con dos pulsaciones, la segunda algo más intensa que la primera, separada por otra de menor entidad más templada, en la que las temperaturas invernales dan lugar a escasos procesos de gelivación.

TD-10.2.- Presenta una potencia de 1,20-1,25 m compuesta de tres niveles bien definidos, todos ellos buzando hacia el centro de la cavidad. Los depósitos basales erosionan profundamente los de la subunidad precedente, llegando en algún punto a alcanzar los de TD-9, clavándose los bloques de aquéllos en éstos.

El nivel inferior, con una potencia de hasta $0,70 \mathrm{~m}$., está compuesto por bloques y cantos 
heterométricos, en todas las posiciones del espacio, englobados en una matriz limoso-arcillosa roja. La matriz arcillosa presenta cierta disyunción prismática deformada por los bloques y un cierto deslizamiento postdeposicional hacia el centro de la depresión. Corresponde a un depósito mixto, en el que mientras se producía la sedimentación de las lutitas aportadas por aguas de escorrentía discontinuas, con etapas de desecación. se producía la caída gravitacional de grandes bloques del techo que se incrustaban o incorporaban al depósito de finos. Después todo el depósito deslizó ligeramente a favor de la pendiente, posiblemente favorecido por la deformación por colapso de los depósitos anteriores en la zona nororiental.

Los bloques corresponden al inicio de la etapa final de desmantelamiento del techo de la cueva. La caída de estos grandes bloques y placas đebe atribuirse a la evolución interior de la cavidad por erosión inversa y exterior por erosión de la vertiente, que dieron lugar al adelgazamiento del techo de la cueva y a la ampliación de fisuras y diaclasas, hasta alcanzar el grado de inestabilidad que provocó su caída. Su atribución a procesos de gelivación no parece suficiente por falta de claros indicadores de climas fríos, aunque no se descarta la posibilidad de que el hielo haya cooperado a estos desplomes, siendo también probable la intervención en estos procesos de las raíces de la vegetación arbórea.

Adaptándose a las irregularidades superficiales del nivel anterior debidas al deslizamiento, o erosionándolo ligeramente, discordante sobre él, se encuentra un nivel intermedio de 0,20 a $0,25 \mathrm{~m}$ de potencia. Está formado por arenas arcillosas y arcillas arenosas según las zonas, con gravas y algunos cantos dispersos y alterados en su interior, masivas y con algunas concreciones calcáreas. A techo se reconoce un horizonte con huesos fósiles. Presenta mayor buzamiento hacia el borde, atenuándose hacia el interior.

Este nivel corresponde a materiales transportados desde la zona externa por procesos de arrollada difusa de carácter esporádico, en un ambiente de clima templado y húmedo.

Sobre el anterior se sitúa el nivel superior de potencia variable hasta $0,30 \mathrm{~m}$. Está compuesto por arcillas rojas masivas, con escasos cantos alterados dispersos en su interior, así como algunos huesos fósiles orientados en el sentido de la estratificación, e incorporando algún bloque caído de mediana talla. Corresponden a aguas de escorrentía de muy baja energía, en un medio en el que prácticamente se mantienen las condiciones climáticas anteriores y si acaso disminuye algo la humedad.

En conjunto esta subunidad representa una amplia fase templada y húmeda, destacando ligeramente la humedad en el tramo intermedio.

TD-10.3.- Potencia 1,30-2,0 m. Esta subunidad se ha separado de la anterior por situarse en discordancia sobre aquélla. Está formada por una secuencia de niveles predominantemente arcillosos rojizos con intercalaciones, discontinuas o en forma de lentejones, de detríticos más gruesos en los que predominan las gravas y gravillas, procedentes todos ellos del entorno exterior. Los niveles detríticos más gruesos presentan algunas nodulizaciones carbonatadas. En estos sedimentos se intercalan grandes bloques de desplomes del techo, que terminan por convertir la antigua cueva en una dolina. Dichos bloques condicionan y modifican localmente y en detalle las áreas de sedimentación en el interior de la depresión. En la zona noroccidental se han distinguido los siguientes niveles de muro a techo:

- Potencia de 0,00-0,20 m. Erosivo sobre la unidad anterior. Formado por gravillas alteradas y escasos cantos hacia la zona central, englobados en una matriz arcillosa roja, con estratificación masiva y deformado por la caída de grandes bloques de orden métrico, previa a la sedimentación del siguiente.

- Potencia 0,30-0,40 m. Arcillas rojas masivas englobando algunas gravas y cantos en la parte superior, sobre todo hacia el centro, donde incluye también algunos bloques. Recubre hacia la zona central parte de los bloques caídos previamente. Contiene fósiles de mamíferos. Recientemente se ha recogido industria lítica del Paleolítico inferior (Mosquera y Carbonell, com. pers.).

- Potencia 0,30-0,60 m. Corresponde a otro nivel de gravillas calcáreas análogo al anterior, pero con mayor proporción de cantos frescos y algún bloque. Contiene huesos fósiles e industria lítica en la zona central de la cubeta.

- Potencia variable en torno a $0,80 \mathrm{~m}$. Compuesto por arcillas rojas masivas, con un cierto tendido en la estratificación, marcado por alineaciones de cantos o niveles de gravas discontinuos.

Estos depósitos se interpretan como aportes procedentes de los bordes de la dolina, trans- 
portados por aguas de escorrentía no canalizadas o pobremente estructuradas. Además de los procesos de hundimiento y colapso acompañantes, se producen aportes gravitacionales debidos a procesos de gelivación. escasos y de escasa intensidad, muy inferior a la registrada en unidades precedentes. Corresponde con un clima "fresco" en el sentido de Hoyos (Hoyos, 1981), con variaciones dentro de un ambiente de humedad estacional parecido al actual.

Entre esta subunidad y la siguiente se produce un último reajuste por colapso de los depósitos preexistentes, con ligera deformación cóncava del fondo de la depresión, lo que facilita la sedimentación de la última unidad.

TD-10.4.- Potencia $0,70-0,80 \mathrm{~m}$. Representan los depósitos más recientes de la zona estudiada del flanco noroccidental de Gran Dolina, sólo cubiertos parcialmente por la unidad terminal TD-11 de Gil et alii (1987). En ella se han distinguido dos niveles.

- El nivel inferior de $0,30 \mathrm{~m}$ de potencia, está formado por arcillas arenosas, masivas o con laminaciones en alguna zona; incluyen hiladas discontinuas de pequeños cantos alterados que marcan el sentido de la estratificación, una más continua, o con más bloques en la base. A techo presenta un lecho de gravillas con la misma matriz, que contiene restos óseos e industria en el contacto y en su interior.

- El nivel superior $0,40-0,50 \mathrm{~m}$ de potencia, es de características parecidas al anterior, sólo que incluye dos alineaciones discontinuas de cantos. A techo está erosionado por la vertiente. Algunas acumulaciones de bloques ocurren en el contacto inferior.

Se interpretan sedimentológicamente como los de la subunidad anterior, salvo que en éstos no aparecen vestigios de gelivación, por lo que se les hace corresponder con un clima templado y más húmedo que el anterior. Las evidencias de encharcamiento y las direcciones de cantos relacionados con lavados laterales, sugieren más humedad que en la subunidad precedente. Abundante fauna e industria del Paleolítico inferior (Aguirre, 1995; Mosquera y Carbonell, 1992).

TD-11.- Todavía por encima de la unidad anterior se encuentra en la zona central de la dolina un conjunto predominantemente arcilloso rosáceo, que engloba algunos bloques, cantos y gravas alterados y dispersos en la matriz y un nivel con grandes placas en la base. Todo el con- junto aparece carbonatado diferencialmente, con nodulizaciones, manchas dispersas y vermiculaciones de carbonatos, debidas a la superposición de procesos edáficos que enmascaran su significado paleoclimático inicial. No se ha estudiado esta parte en detalle.

\section{DATOS CRONOLÓGICOS}

En la parte inferior del corte de Gran Dolina (TD), la polaridad magnética inversa dentro y cerca de la base de la unidad TD-3, indica que estas unidades inferiores preceden al evento magnético Matuyama/Brunhes (Carracedo, Soler y Chicharro, com. pers.). Ello es coherente con las evidencias geodinámicas según Zazo et alli (1983), del cambio a karst freático maduro entre las terrazas 3 y 4 del Arlanzón, del Pleistoceno Inferior y con las faunísticas (Aguirre, 1995). La fecha de la inversión Matuyama/Brunhes varía según los autores. Se puede retener como aproximación segura una banda entre 740.000 y poco más de 770.000 años antes del Actual. La serie de depósitos de la Trinchera habría comenzado, según ese dato, hace más de 750.000 años con seguridad, pero no mucho más, al final del Pleistoceno Inferior.

Se pueden atribuir datos de edad a los niveles intermedios de Gran Dolina, con calibración fiable, por interpolación entre los datos mencionados arriba, controlada por correlación paleofaunística con yacimientos de Europa directamente datados (Aguirre, 1995). Así podemos asegurar una edad superior a $510 \mathrm{ka}$-la edad de Belle-Roche y Mauer para TD6, nivel con los recientes restos de fósiles humanos aparecidos en 1994 , y 300/325 ka para TD10, esto es no más moderna que Castel di Guido y Bilzingsleben. Los niveles intermedios TD7, TD8 y TD9 tendrían correspondencia predecible con las fluctuaciones climáticas de entre hace 500 y $350 \mathrm{ka}$.

\section{FLUCTUACIONES TÉRMICAS EN GRAN DOLINA Y EPISODIOS ISOTÓ- PICOS OCEÁNICOS}

Entre los datos basal y terminal de la secuencia de depósitos de Atapuerca, poco más de 800 ka B.P. y cerca de 200 ka B.P., respectivamente, se puede trazar una curva relativa y cualitativa de ciclos mayores de calor y frío a lo largo de la 
secuencia Gran Dolina. El número de ciclos principales es de 6 episodios dominantemente cálidos o templados (TD-3, TD-5.1, TD-5.3. TD7. TD-8.4/TD-9, TD-10.2 a 10.4) y otros tantos, marcados por uno o más picos fríos (TD-2, TD4. TD-5.2, TD-6, TD-8.1 a TD-8.3 y TD-10.1). Dada la duración total de la secuencia, algo más de 550.000 años, cada ciclo completo - frío y cálido- representado aunque de modo discontinuo por dichas unidades sedimentarias y sus alteraciones epigenéticas, habría durado más de 80.000 años como media. La duración media es equivalente a la de los ciclos isotópicos del océano basados en las relaciones de oxígeno $16 / 18$. Teniendo en cuenta que podría faltar el registro de un ciclo climático principal -difícilmente más de uno- en hiato sedimentario, no es improbable que tengamos el mismo número de ciclos calientes y fríos registrados en la secuencia sedimentaria del karst de Atapuerca que en el océano, entre las fechas que enmarcan el Pleistoceno Medio. Lo más probable es que coincidan los unos con los otros y se pueda dar como bastante segura su equivalencia, según se muestra en la figura 4 de Aguirre y Hoyos (1992), y en la figura 2 de este trabajo.

\section{VARIACIONES DE HUMEDAD EN EL PLEISTOCENO MEDIO SEGÚN EL REGISTRO DE GRAN DOLINA}

Los gradientes de humedad en relación con los distintos ciclos cálidos y fríos en esta parte de España y a lo largo del Pleistoceno Medio, tal como se registran en el corte de TD en la Trinchera de Atapuerca, no son siempre iguales.

Se entiende en este trabajo con el adjetivo «estacional» el hecho de que las etapas de lluvia anuales, una o dos, están separadas por estaciones más secas, independientemente de la pluviosidad total, que define el carácter básicamente húmedo o seco del clima.

En general, los episodios cálidos tal como se reflejan en la serie de depósitos de Gran Dolina, comienzan con humedad alta o en aumento, pero con diferente tendencia y evolución. Así, al final de TD-2 la humedad decrece y vuelve a aumentar discretamente, siempre estacional en TD-3. Entre TD-6 y TD-7, la humedad que ha venido creciendo causa una fuerte erosión; la precipitación es estacional y alta en la primera mitad de TD-7, decayendo en la segunda mitad.
A lo largo de TD-8 aumenta considerablemente la humedad, con mejoría térmica al final. Después de TD-8 se registra una fuerte erosión; cae la humedad y el clima pasa a seco, y torna a hacerse medianamente húmedo en TD-9. En el episodio cálido representado en TD-10.2 a TD10.4, e intervalo hasta la base de TD-11, se parte de condiciones de baja humedad, y se continúa con una humedad creciente con oscilaciones menores y carácter estacional, produciendo efectos erosivos y deslizamientos, con mayor intensidad al final. Dos casos netamente inversos se presentan en las fluctuaciones de TD-5.1 y TD-5.3: en la primera, fresca y templada, hay una acusada estacionalidad de lluvias inicialmente que decrecen sensiblemente con oscilaciones, presentando una ligera recuperación al final (antes y en los rigores fríos de TD-5.2); en el caso TD-5.3, el clima es cálido y fuertemente estacional, con precipitaciones escasas frente a una larga estación estival, si bien con una oscilación en medio debida a un aumento transitorio de la humedad, que se llega a recuperar con los primeros fríos que siguen (Fig. 2).

En los episodios fríos registrados en Gran Dolina también varía de diverso modo la humedad. Un aumento decidido de la humedad a lo largo del episodio frío, aun cuando con inflexiones menores, se observa en nuestros niveles TD4 . TD-6 y TD-8. Una oscilación regular en la humedad, que viene a ser mayor que la de los dos períodos cálidos precedente y siguiente, se aprecia en TD-5.2, y una caída considerable de la humedad, aunque breve, en TD-10.1.

Las variaciones de humedad a lo largo del Pleistoceno Medio que inferimos del carácter de los tramos sedimentarios que se suceden en la exposición del corte TD de Gran Dolina muestran, pues, lo primero unas oscilaciones o ciclos que no coinciden con los ciclos de temperatura, como hemos señalado en otro trabajo (Aguirre y Hoyos, 1992). Se ha de concluir por ello que para construir los modelos de cambio climático se ha de tener en cuenta, en latitudes medias, no sólo las variables orbitarias que afectan a la insolación y al deshielo y se reflejan en las temperaturas del agua oceánica, sino además las modificaciones en la circulación atmosférica que se relacionan con la distribución de la humedad en general y con el régimen de lluvias, y sus gradientes regionales. Estas variables pueden estar condicionadas por factores geomorfológicos añadidos a las variaciones térmicas en los océa- 
nos y a los presumibles efectos aditivos y de realimentación en los ciclos de variables orbitarias y de glaciación.

En segundo lugar, observamos otras irregularidades en los ciclos de humedad: Una es su duración o frecuencia. Los máximos de humedad registrados en TD se pueden datar. de acuerdo con las correlaciones que proponemos: en torno a $700 \mathrm{ka}$, hacia 520 y $420 \mathrm{ka}$, y cerca de $330 \mathrm{ka}$. Los máximos de aridez están sobre 660 ka, 600-580 ka, 460 ka, 400 ka, 360 ka, y menos de 190 ka. Por análisis palinológico sobre muestras de sondeo en plataforma continental africana, en el Atlántico oriental cerca del trópico de Cáncer, se han señalado varias crisis de aridificación cerca de $580 \mathrm{ka}$, en 480 y $470 \mathrm{ka}$, cerca de $400 \mathrm{ka}$ y de $360 \mathrm{ka}$, y recuperaciones de humedad cerca de 340 ka y de 240 ka (Dupont, 1992; v. Fig. 4). Estas crisis pueden compararse con las obtenidas con nuestras inferencias y datadas indirectamente por correlación con los episodios oceánicos (Aguirre, 1995).

\section{BIBLIOGRAFÍA}

Aguirre, E. (1992): «Atapuerca land change, caves and humans over Middle Pleistocene». Journal of Human Ecology, 2 (3) 3 (1): 227-270.

- (1994): «Dating the Ibeas Humans: Attempts and Question Marks». Courier Forshungs-Ins. Senckenberg, 171: 197-204.

- (1995): «Atapuerca (Burgos, España): Su contribución a las ciencias del Cuaternarion. Revista Española de Paleontología, 10 (1): 58-82.

Aguirre. E. and Hoyos, M. (1992): "Climate record in cave deposits: the Atapuerca TD case». In G.J. Kukla
\& E. Went (cds.): Start of a (ilacial. Springer Verlag (NATO. ASI. S.r. 1.3). Berlin: 127-136.

DI poN1. L. M. (1992): "Marine palynology of interglacialglacial transitions". In (i.J. Kukla \& F. Went (eds.): Start of a (ilacial. Springer Verlag. (NATO ASI. Ser. 1.3). Berlin: 1.37-156.

Gil.. E.: Agit IRre. E. \& Hoyos, M. (1987): "Contexto estratigráficom. En E. Aguirre. E. Carbonell \& J.M. Bermúdez de Castro (eds.): El hombre fósil de lheas y el Pleistocenos de la Siera de Atupuerca. Junta de Castilla y León. Valladolid: 47-54.

Hoyos. M. (1979): El kurst en Asturias durante el Pleistoceno superior y Holoceno: Geromorfologia. Sedimentologi. Paleoclimatologia. Tesis Doctoral. Universidad Complutense de Madrid.

- (1981): «Estudio geológico de la Cueva de Las Caldas: Geomorfología, sedimentología e interpretación paleoclimática». En Mª. S. Corchón: La Cueva de Las Caldas, San Juan de Priorio (Oviedo). Excavaciones Arqueológicas en España. Ministerio de Cultura. Madrid. Memoria 115: 23-63.

Hoyos, M.; Bustillo, M. A.; García, A.; Martín EscorZA, C.: ORTIz R. \& SuAzo, C. (1982): "Caracteristicas geológico-kársticas de la cueva de Altamira». Informe Ministerio de Cultura. Subd. General Arqueología. Madrid.

LlopIS Lladó. N. (1970): «Fundamentos de Hidrogeología cárstica». Blume. Barcelona.

Martín Merino, M. A.: Domingio Mena, S. \& Antón PalAcios, T. (1981): "Estudio de las cavidades de la zona BU-IV-A (Sierra de Atapuerca)». Kaite, 2: 41-76. Burgos.

Mosquera, M. y CARBonell, M. (1992): «La talla lítica en Atapuerca (Burgos)». Trabajos de Prehistoria, 49: 131154.

Zazo, C.; Goy, J.L. \& Hoyos, M. (1983): «Estudio geomorfológico de los alrededores de la Sierra de Atapuerca (Burgos)». Estudios Geológicos, 39: 179-185. 